\title{
Quantum efficiency, intrinsic emittance, and response time measurements of a titanium nitride photocathode
}

\author{
Chenjie An, ${ }^{1}$ Rui Zhu $\odot,{ }^{1,}{ }^{*}$ Jun $\mathrm{Xu},{ }^{1}$ Yaqi Liu, ${ }^{1}$ and Dapeng $\mathrm{Yu}^{2,1}$ \\ ${ }^{1}$ Electron Microscopy Laboratory, School of Physics, Peking University, Beijing 100871, China \\ ${ }^{2}$ Shenzhen Institute for Quantum Science and Engineering, and Department of Physics, \\ Southern University of Science and Technology, Shenzhen 518055, China
}

(Received 15 November 2020; revised 20 July 2021; accepted 23 August 2021; published 14 September 2021)

\begin{abstract}
The photoemission properties of photocathodes affect the ultimate performance of electron accelerator based facilities and the cost. Several major properties of titanium nitride (TiN) irradiated by ultraviolet femtosecond laser pulses were studied. Specifically, the quantum efficiency and intrinsic emittance were measured, and the response time was estimated indirectly. For photon energies higher than the work function, quantum efficiency values of $7 \times 10^{-8}-5 \times 10^{-6}$ were obtained. The measured intrinsic emittance was $0.54 \mu \mathrm{m} / \mathrm{mm}$ for the typical wavelength of $266 \mathrm{~nm}(4.66 \mathrm{eV})$. When the photon energy was lower than the work function, two-photon photoemission appeared, and the intrinsic emittance increased with the laser intensity. At photon energies of higher than and slightly lower than the work function, the intrinsic emittance ranged from 0.28 to $0.60 \mu \mathrm{m} / \mathrm{mm}$ and tended toward a minimum constant of $0.28 \mu \mathrm{m} / \mathrm{mm}$ if only single-photon photoemission was considered. The response time of TiN was proven to be close to that of gold by comparing the transverse expansion of electron beams under space charge force for the irradiated photon energy higher than the work function. The autocorrelation measurement of the photoemission of TiN and gold were performed, and a comparison of these results indicated that the two-photon photoemission of TiN excited by a photon energy slightly lower than the work function was prompt.
\end{abstract}

DOI: 10.1103/PhysRevAccelBeams.24.093401

\section{INTRODUCTION}

Photoemission based electron sources are widely used in cutting-edge applications of electron accelerators, such as ultrafast electron diffraction (UED) [1,2], x-ray free electron lasers (FEL) [3], and inverse Compton scattering based $\mathrm{x}$-ray sources [4]. As a crucial component of the electron source, the properties of the photocathode affect the quality of the electron beam and thus the ultimate performance and the cost of these facilities [4-6].

The main properties of a photocathode are the response spectrum, quantum efficiency, intrinsic emittance, response time, operational lifetime, vacuum requirement, etc., [7]. An ideal photocathode usually has high quantum efficiency, small intrinsic emittance, fast response time, and a long operational lifetime [8,9]. However, there is no photocathode that meets all these criteria simultaneously, and consequently the selection of the material depends mainly on the required

\footnotetext{
"zhurui@pku.edu.cn

Published by the American Physical Society under the terms of the Creative Commons Attribution 4.0 International license. Further distribution of this work must maintain attribution to the author(s) and the published article's title, journal citation, and DOI.
}

properties. Many different photocathode materials have been developed to meet these practical needs.

There are two types of main photocathode materials: metals and semiconductors [9]. Metals are characterized by small intrinsic emittance, fast response time, a long operational lifetime, and low requirement for operational vacuum. However, the response spectrum is in the ultraviolet range, and their quantum efficiency is often low. In contrast, semiconductors have high quantum efficiency, and the response spectrum can be in the visible range; but the response time is usually longer, the environmental vacuum requirement is extremely high, and the operational lifetime is short [7-12].

Increasing the photon energy is an effective way to heighten the quantum efficiency of the photocathode, but it also induces unwanted growth of intrinsic emittance [13]. In recent years, both increasing the electron yield of metal cathodes and simplifying the laser system by nonlinear photoemission have been studied [14-17], that is, directly exciting the cathode with infrared or visible laser, which bypasses some inefficient optical frequency up-conversion. However, antireflection film [14] or a surface plasma structure $[15,16]$ is needed to enhance absorption because of the high reflectivity of metals in this wavelength range. With a carefully designed surface structure, the reflectivity can be less than 1\% [16]. Besides photon energy, surface 
roughness [18] and cathode temperature [19] can also affect intrinsic emittance. By improving the surface roughness or cooling the photocathode, the intrinsic emittance can be reduced.

TiN is a kind of ceramic material with a high electric conductivity comparable to metals. The carrier concentration of TiN is on the order of $10^{22} \mathrm{~cm}^{-3}$ [20], which is close to that of copper and silver. The work function of TiN reported in the literature ranges from 4.14 to $4.87 \mathrm{eV}$ $[21,22]$, corresponding to photon energy in the ultraviolet range. Several properties of TiN suggest that it may have excellent performance as a photocathode. TiN is an alternative plasmonic material similar to gold, and, as a nonstoichiometric material, the optical properties can be tuned by varying the composition [23-25]. At present, there is mature technology for the micromachining and nanomachining of TiN [26]. Thus, it is possible to obtain higher electron gain by nonlinear photoemission. Single crystal TiN films can be prepared by molecular-beam epitaxy technology [27], so that the uniform smooth surface can inhibit the intrinsic emittance growth caused by the physical and chemical roughness of the surface. In the ultraviolet to near-infrared regions, the optical penetration depth of TiN is in the range of 10-40 nm [28,29], which means that the photoemission may have a relatively short response time. TiN has a high melting point, high hardness, and good chemical stability, and is often used as a hard coating protective film. This shows that TiN does not require a high vacuum in the working environment and may have a long operational lifetime. What is more, high quality TiN has a relatively high critical temperature of around $5 \mathrm{~K}$ [30], which indicates its possible application in superconducting rf photocathode guns [31]. In this paper, the quantum efficiency, intrinsic emittance, and response time of TiN film excited by ultraviolet light are characterized, and the feasibility of using TiN as a new type of photocathode material is considered.

\section{EXPERIMENTAL SETUP AND MEASUREMENT METHODS}

\section{A. Photocathodes}

The TiN film with a thickness of $100 \mathrm{~nm}$ was deposited on a (111)-oriented silicon substrate by argon ions sputtering deposition with a precision etching and coating system (Gatan 682 PECS). The substrate was cleaned successively by acetone, ethanol, and deionized water with an ultrasonic cleaner. Then, the substrate was dried by blowing with high pressure nitrogen and loaded into the vacuum chamber. A TiN target with a purity of $99.99 \%$ was used. The chamber was pumped down to a base pressure of $4 \times 10^{-5} \mathrm{~Pa}$ before the argon gas was injected. During the deposition, the pressure of the chamber was kept at $0.01 \mathrm{~Pa}$. The deposition rate was approximately $3.3 \mathrm{~nm} / \mathrm{min}$. After the deposition, the sample was pasted on a copper sample holder with ultrahigh vacuum (UHV) compatible silver loaded epoxy and heated to $150^{\circ} \mathrm{C}$ for an hour in high vacuum to cure the binder, and then transported to a UHV chamber for subsequent measurement. Surface morphology of the TiN film was measured using atomic force microscopy (Bruker Dimension Icon). The surface root-meansquare (rms) roughness of the photocathode is about $0.5 \mathrm{~nm}$. See the Appendix A for an AFM image of the TiN photocathode.

In order to study the response time of the TiN film, a $100 \mathrm{~nm}$ thick gold film was also prepared as a control. The preparation conditions and processes for the gold film were the same as that of the TiN, except that a target of $99.99 \%$ of pure gold was used. The deposition rate was approximately $7.1 \mathrm{~nm} / \mathrm{min}$.

\section{B. Laser system}

The ultrashort laser pulses used in our experiment were generated by a wavelength tunable Ti: sapphire infrared laser (Spectra-Physics Tsunami 3960C-25XP)-from which the pulse duration was less than 100 fs FWHM and the repetition rate was $80 \mathrm{MHz}$ - and a third harmonic generator (SpectraPhysics GWU-UHG-23FS) system. The laser was focused to an approximately $100 \mu \mathrm{m}$ FWHM spot onto the cathode at an incidence angle of $35^{\circ}$. During the measurement, UV fused silica reflective neutral density filters were used to adjust the laser power to meet the requirements of the beam current. The incident light used in the entire experiment was p-polarized unless otherwise specified.

\section{Intrinsic emittance measurement}

The characterization of the TiN photocathode was completed on our self-built photocathode test system [32]. Solely the scintillator screen was replaced with a microchannel plate (MCP) and fluorescent screen module to facilitate measurement with much lower beam currents. This system was built to measure the intrinsic emittance of a photocathode based on the free expansion method $[33,34]$. The photoelectrons are accelerated only along the direction perpendicular to the surface of the photocathode before passing through the metal grid in the anode arranged parallel to the cathode and drift in a field free region to a scintillator screen or an $\mathrm{MCP}$ and fluorescent screen module where the beam is imaged. The relationship between the transverse momentum $p_{x}$ (in the $x$ direction) of an electron that remains constant during the drift and its displacement $x$ from the center of the spot in the image is as follows [32]:

$$
p_{x}=\frac{x}{2 g+d} \sqrt{2 m_{e} e V},
$$

where $g$ is the gap between the photocathode and the grid, $d$ is the distance from the grid to the scintillator screen or the MCP surface, $m_{e}$ and $e$ are the electron rest mass and 
the electron charge, respectively, and $V$ is the absolute value of the potential applied on the cathode which is set generally at $5 \mathrm{kV}$. Thus, the transverse momentum distribution and hence the transverse intrinsic emittance can be obtained from the image of the electron beam. The double Gaussian function mentioned in Ref. [32] was more suitable for fitting the transverse momentum distribution containing two-photon photoemission. In this article, according to the transverse momentum distribution function of polycrystalline cathode presented in Ref. [35],

$$
\begin{aligned}
G_{\text {Spot }}= & A\left(L i_{2}\left\{-\exp \left[E\left(C-\frac{p_{x}^{2}}{2 m_{e} e}\right)\right]\right\}\right. \\
& \left.-L i_{2}\left\{-\exp \left[E\left(C-D-\frac{p_{x}^{2}}{2 m_{e} e}\right)\right]\right\}\right)+B
\end{aligned}
$$

was used to fit the single-photon photoemission data. In the above equation, $G_{\text {Spot }}$ represents the gray values of the image corresponding to $p_{x}$, the fitting parameter $A$ is related to the specific gray values of the image, and $B$ represents the uniform background of the images. The rms of the transverse momentum can be expressed by the fitting parameters $C, D$, and $E$ as

$$
\sqrt{\left\langle p_{x}^{2}\right\rangle}=\sqrt{\frac{m_{e} e}{E} \frac{L i_{4}[-\exp (C E)]-L i_{4}\{-\exp [(C-D) E]\}}{L i_{3}[-\exp (C E)]-L i_{3}\{-\exp [(C-D) E]\}}} .
$$

The polylogarithm functions in Eqs. (2) and (3) are defined as [35]

$$
L i_{n}(z)=\frac{(-1)^{n-1}}{(n-2) !} \int_{0}^{1} \frac{1}{t}[\ln (t)]^{n-2} \ln (1-z t) d t .
$$

If the beam current used in the intrinsic emittance measurement is too large, the expansion of electron bunches caused by Coulomb force among the electrons will increase the cross section of the electron beam on the detector and make the measured intrinsic emittance larger. To avoid the influence of Coulomb force, very low beam currents were used to ensure the average electron numbers per pulse were kept at less than 1 . When the photon energies were near to or lower than the work function, much lower beam currents were used in order to suppress the nonlinear process.

\section{Quantum efficiency measurement}

Quantum efficiency, defined as the number of emitted electrons per incident photon, was obtained by recording the photocurrent with a high precision source meter (Keithley 6487) applying a $-500 \mathrm{~V}$ potential on the cathode and measuring the power of light incident onto the cathode surface. To measure the light power on the cathode, a small fraction of the input laser beam was split off and monitored with a power meter before the laser entered the vacuum chamber.

\section{E. Response time estimation}

The apparatus described above was not designed to directly measure the length of electron bunches or the response time of the photocathode. However, it could be used to estimate the response time of a cathode indirectly. Different than the low beam current setting-less than one electron in a pulse on average-in emittance measurement, if a large beam current is used, the Coulomb force will cause an expansion of the beam bunches and enlarge the fluorescent spot. The intensity of the Coulomb force is determined by the spatial density distribution of the electrons in a bunch. Assuming the bunch charge is constant, the intensity of the Coulomb force will depend primarily on the bunch length if the phase space density distribution at the photocathode is controlled. The phase space density distribution is codetermined by the incident laser spot size and the intrinsic emittance. The beam length is affected by the laser pulse duration, the response time of the photocathode, and the accelerating voltage between the electrodes. Setting the bunch charge, the incident laser spot size, intrinsic emittance, laser pulse duration, and the accelerating voltage between the electrodes as constants, the size of the fluorescent spot will be determined by the response time of the photocathode. Conversely, the response time of the photocathode can be estimated by measuring the size of the fluorescent spot. Due to the complex relationship between the photocathode response time and the size of the fluorescent spot, there is no analytical expression, and an accurate response time measurement requires numerical simulation. It is well known that metals have a response time of tens of femtoseconds. Thus, we can compare the response time of TiN and gold by comparing the expansion of the beam bunches caused by Coulomb force. In addition to using large beam current, to make the fluorescent spot size on the screen depend solely on the response time of the photocathode, the following items need to be controlled in the TiN and gold experiment: (1) the same accelerating voltage between electrodes (3 kV was used) must be chosen; (2) a suitable laser wavelength at which the intrinsic emittance of TiN and gold are approximately equal must be chosen; (3) the laser spot size on the cathodes must be consistent; and (4) and the same laser pulse duration must be selected.

The autocorrelation technique can be used to estimate the response time of the photoemission containing nonlinear processes when the photocathode is irradiated by a long wavelength laser pulse. A basic time delay optical path for second-order autocorrelation is analogous to that of a Michelson type autocorrelator [36-38] and is sketched in Fig. 1. A nonpolarizing beam splitter splits the input laser pulse into two replicas, which are then retroreflected by a 


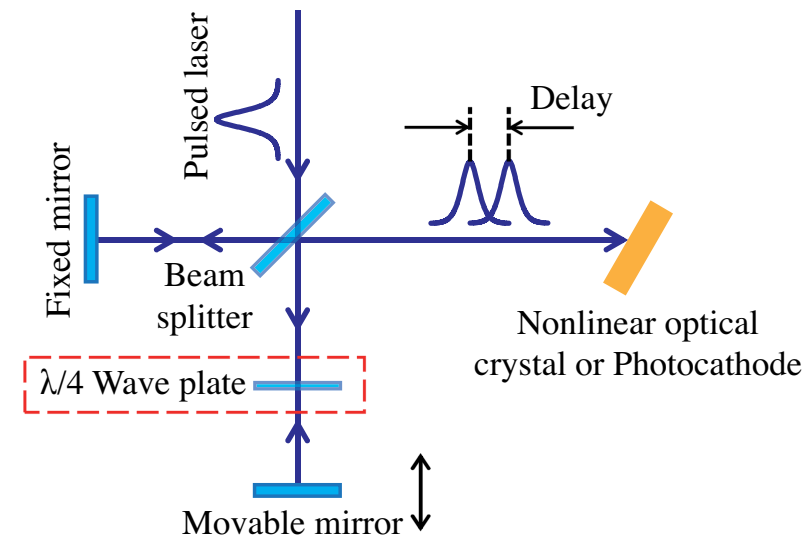

FIG. 1. Schematic of the time delay optical path for an autocorrelation measurement. The incident laser pulse is divided into two pulses with a certain time delay and then are incident to a nonlinear optical crystal or a photocathode. The time delay is adjusted by moving the movable mirror back and forth. The $\lambda / 4$ wave plate is used to rotate the polarization of the beam passing through it twice by $90^{\circ}$.

fixed or a movable mirror and recombined by the same splitter. After that the original pulse is transformed into two collinear pulses incident to a nonlinear optical crystal or a photocathode with a time delay. The autocorrelation signal induced by the combined beam as a function of the time delay can be used to calculate the laser pulse duration. The $\lambda / 4$ wave plate is added to rotate the polarization of the beam passing through it twice by $90^{\circ}$, thus to switch the interference autocorrelation measurement to intensity autocorrelation measurement. If the temporal response of a photocathode is prompt, the laser pulse duration measured with the photocathode should be very close to the actual duration of the incident pulse utilizing two-photon photoemission photocurrent as the second-order autocorrelation signal $[39,40]$. Otherwise, the measured values will be larger than the actual duration. Assuming that the third harmonic laser used in the experiment is a $\operatorname{sech}^{2}$-shaped pulse laser, the pulse duration can be extracted by fitting the intensity autocorrelation signal or the average of the upper and lower envelope of the interferometric autocorrelation signal as a function of time delay with this expression:

$$
\begin{aligned}
& I_{\text {Current }}(\tau) \\
& =A \frac{\frac{1.763\left(\tau-\tau_{0}\right)}{\tau_{p}} \cosh \left(\frac{1.763\left(\tau-\tau_{0}\right)}{\tau_{p}}\right)-\sinh \left(\frac{1.763\left(\tau-\tau_{0}\right)}{\tau_{p}}\right)}{\left[\sinh \left(\frac{1.763\left(\tau-\tau_{0}\right)}{\tau_{p}}\right)\right]^{3}}+B,
\end{aligned}
$$

where $\tau_{p}$ is the laser pulse duration (FWHM), $\tau-\tau_{0}$ is the time delay, and when $\tau=\tau_{0}$, the two optical pulses coincide. See Appendix B for the detailed derivation of Eq. (5).

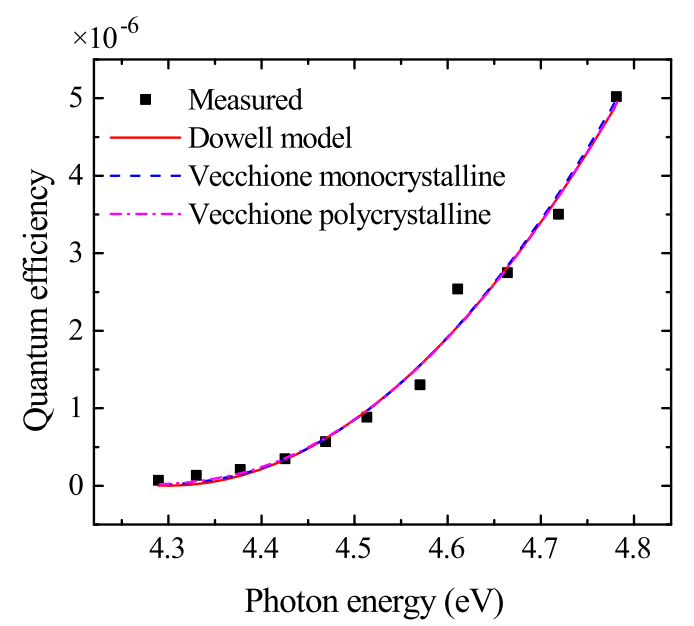

FIG. 2. Photon energy dependence of quantum efficiency. The work functions obtained from fitting with the Dowell model [13] and Vecchione monocrystalline model [35] are 4.30 and $4.31 \mathrm{eV}$, respectively. From the Vecchione polycrystalline model [35] they are $4.26-4.36 \mathrm{eV}$.

\section{RESULTS AND DISCUSSION}

The dependence of the quantum efficiency on the photon energy is shown in Fig. 2. The quantum efficiency of TiN was in the range of $7.0 \times 10^{-8}-5.0 \times 10^{-6}$ for the photon energy of 4.29-4.78 eV. For a typical wavelength of $266 \mathrm{~nm}(4.66 \mathrm{eV})$, the photocurrent density as a function of the laser intensity is shown in Fig. 3. The quantum efficiency fitted from these data was $2.8 \times 10^{-6}$, lower than that of copper [41-43] and magnesium [44-46], which are commonly used in the rf photocathode gun and the dc electron gun. The red solid curve and blue dashed curve in Fig. 2 are fitted according to the Dowell model [13] and the Vecchione monocrystalline model [35], and the work functions are correspondingly 4.30 and $4.31 \mathrm{eV}$. There is

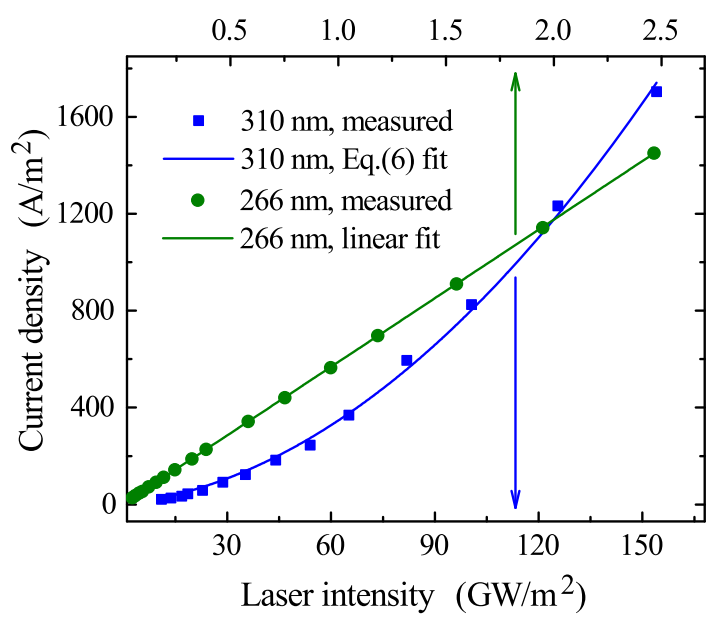

FIG. 3. Current density, charge of a single pulse divided by the product of the laser spot area and the pulse duration (FWHM), as a function of laser intensity for the wavelength of $266 \mathrm{~nm}$ $(4.66 \mathrm{eV})$ and $310 \mathrm{~nm}(4.00 \mathrm{eV})$. 
almost no difference between the Vecchione polycrystalline fit and the former two. The magenta dotted dashed curve indicates the Vecchione polycrystalline fit result with the work functions in the range of 4.26-4.36 eV, which agrees with the published 4.14-4.87 eV [21,22]. Low quantum efficiency necessitates the use of high fluence lasers, which limits the application of TiN. Because the quantum efficiency of a photocathode is related to surface adsorption, the fact that our sample had never undergone any surface treatment after deposition may be the reason for such a low quantum efficiency. Surface cleaning, such as laser cleaning [47], hydrogen ion beam bombardment [48], or argon ion beam bombardment [49], should be performed to improve quantum efficiency.

When driven by photons with the energy slightly lower than the work function, such as the $310 \mathrm{~nm}(4.00 \mathrm{eV})$ case in Fig. 3, the photocurrent density does not change linearly with the laser intensity, which means that the photoemission contained a nonlinear process. These data were well fitted by a straight line with a slope of 1.71 in double logarithmic coordinates as shown in Fig. 4. The noninteger slope indicates that the process is not a pure n-photon photoemission. While in the linear coordinates, Fig. 3, these data were best fitted by a quadratic function with an ordinate intercept of zero,

$$
J=\sigma_{1} I+\sigma_{2} I^{2},
$$

where $\sigma_{n}$ is the generalized $\mathrm{n}$-photon ionization cross section as a function of the photon energy, the electronic temperature, and the properties of the photocathode. Quadratic function fitting indicated that the photoemission contained single- and two-photon photoemission, which was similar to our work about copper cathodes [32].

The intrinsic emittance of TiN film as a function of the photon energy is shown in Fig. 5. The measured intrinsic emittance was in the range of $0.28-0.6 \mu \mathrm{m} / \mathrm{mm}$. When

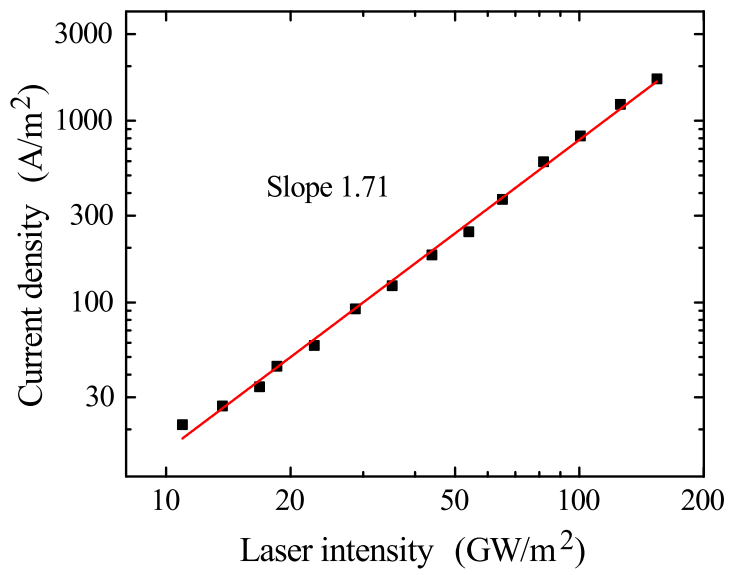

FIG. 4. Current density as a function of laser intensity for $310 \mathrm{~nm}(4.00 \mathrm{eV})$ in the log-log scale. These data are well fitted by a straight line with a slope of 1.71 .

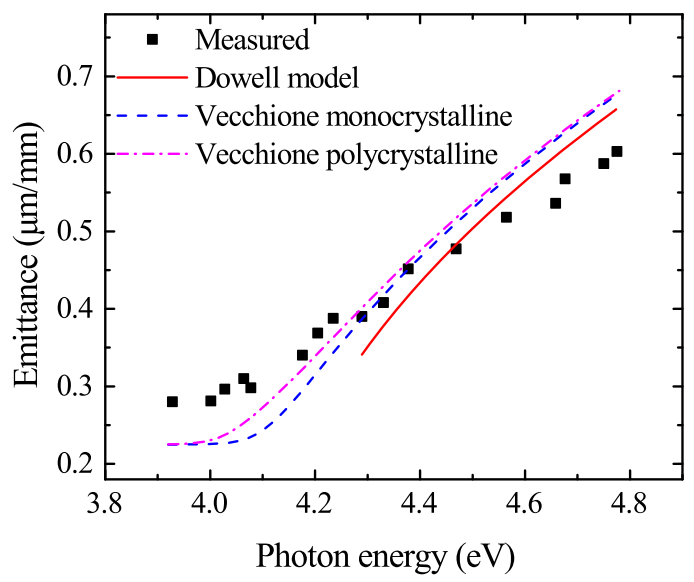

FIG. 5. Measured transverse emittance of TiN film as a function of the photon energies. The work functions obtained from fitting with the Dowell model [13] and the Vecchione monocrystalline model [35] are 4.11 and $4.08 \mathrm{eV}$, respectively; and from the Vecchione polycrystalline model fit [35] the work functions are in the range of $4.00-4.16 \mathrm{eV}$.

irradiated by photons with higher energy, the intrinsic emittance was close to that of metals $[41,43,45]$. For a typical wavelength of $266 \mathrm{~nm}(4.66 \mathrm{eV})$, the emittance was $0.54 \mu \mathrm{m} / \mathrm{mm}$. However, in the case of lower photon energy excitation, emittance increased with laser intensity due to the containing of two-photon photoemission, as shown in Fig. 6. Here we took the asymptotic value at low laser intensity as the emittance in Fig. 5. The intrinsic emittance tends to a constant value of $0.28 \mu \mathrm{m} / \mathrm{mm}$ when the photon energy was low enough. This trend was consistent with the Vecchione model [35] and the Feng model [50], and the asymptotic value of the small photon energy side was also close to the room temperature limit of $0.23 \mu \mathrm{m} / \mathrm{mm}$ as described in the literature. The intrinsic emittance values

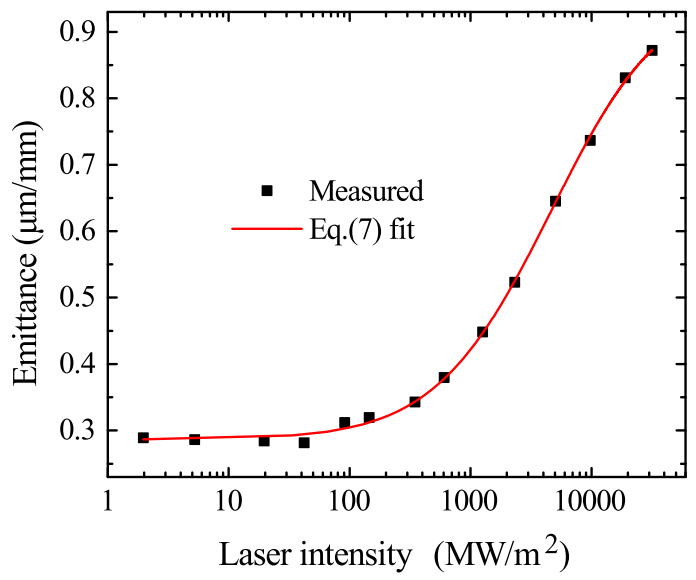

FIG. 6. Laser intensity dependence of measured emittance for $310 \mathrm{~nm}(4.00 \mathrm{eV})$. The average electron numbers per pulse were about 0.81 for the maximal laser intensity. Fitted with Eq. (7), the emittance is $0.29 \mu \mathrm{m} / \mathrm{mm}$ for single-photon photoemission and $0.96 \mu \mathrm{m} / \mathrm{mm}$ for two-photon photoemission. 
calculated from the Dowell model [13] and the Vecchione model were also plotted in Fig. 5. In order to bring the calculated values closer to the experimental data, the effective work functions corresponding to the Dowell model and the Vecchione monocrystalline model were 4.11 and $4.08 \mathrm{eV}$, respectively; and to the Vecchione polycrystalline model the work functions were $4.00-4.16 \mathrm{eV}$. The measured intrinsic emittance was consistent with the theoretical model in terms of the change trend as a function of photon energy, while the values were somewhat different. Furthermore, the work function used here was slightly lower than that obtained from the quantum efficiency. These differences may be related to the morphology of the cathode surface and experimental errors. In addition, these theoretical models were based on some simplifications and approximations, which may account for the difference between the experimental values and the theoretical models.

The increase of intrinsic emittance with laser intensity in Fig. 6 can be explained by the containing of twophoton photoemission. The total photocurrent density $J$ is expressed as a quadratic function of laserintensity $I$, Eq. (6). If we assume that the emittance of single-photon photoemission is $\varepsilon_{1}$, and that of two-photon photoemission is $\varepsilon_{2}$, and $\varepsilon_{1}<\varepsilon_{2}$, then the total emittance can be expressed as

$$
\begin{aligned}
\varepsilon & =\sqrt{\frac{\sigma_{1}}{\sigma_{1}+\sigma_{2} I} \varepsilon_{1}^{2}+\frac{\sigma_{2} I}{\sigma_{1}+\sigma_{2} I} \varepsilon_{2}^{2}} \\
& =\sqrt{\frac{\sigma_{1}}{\sigma_{1}+\sigma_{2} I}\left(\varepsilon_{1}^{2}-\varepsilon_{2}^{2}\right)+\varepsilon_{2}^{2}} .
\end{aligned}
$$

Obviously, the total emittance increases with laser intensity. The solid red curve in Fig. 6 is fitted by Eq. (7), and the fitted $\varepsilon_{1}$ and $\varepsilon_{2}$ were 0.29 and $0.96 \mu \mathrm{m} / \mathrm{mm}$, respectively.

The fluorescent spot size for gold and TiN at the wavelengths of around $260 \mathrm{~nm}$ as a function of the average number of electrons per pulse (charge of the bunch) is shown in Fig. 7. With the increase of the number of electrons, the expansion of the fluorescent spots corresponding to the gold and TiN cathodes were very similar, which indicated that the response time of TiN was very close to that of gold. Therefore, we believe that the photoemission of TiN is prompt.

The number of electrons in Fig. 7 ranges from dozens to more than 10000. Accordingly, the laser intensity on the photocathode changed from a few tenths to several hundred $\mathrm{GW} / \mathrm{m}^{2}$. In order to eliminate multiphoton photoemission, which leads to a larger emittance and hence a larger fluorescent spot, we recorded the dependence of the electrons number on the photons number during the measurement of the fluorescent spot and then made a linear fitting in the double logarithmic coordinates. The fitted slopes corresponding to the three measurements in Fig. 7 were $0.999,1.004$, and 0.999 , which indicated that the photoemission did not contain nonlinear effects.

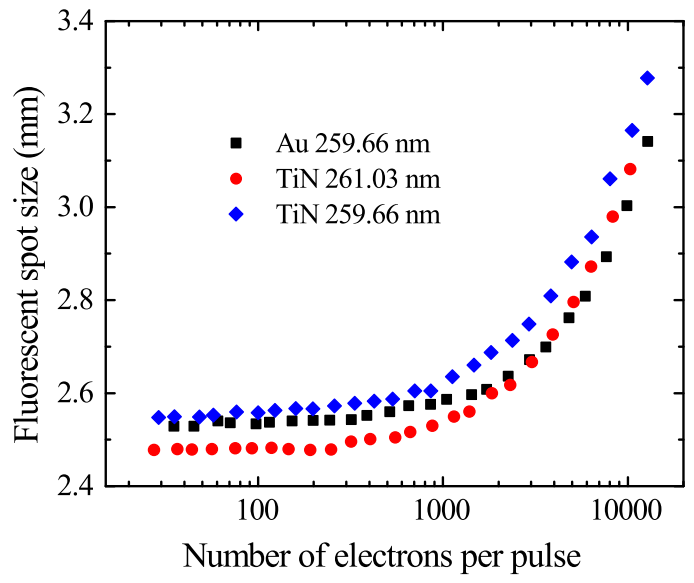

FIG. 7. Root-mean-square fluorescent spot size for $\mathrm{Au}$ (at laser wavelength of $259.66 \mathrm{~nm}$ ) and TiN (at laser wavelengths of 261.03 and $259.66 \mathrm{~nm}$ ) as a function of the average number of electrons per pulse. The quantum efficiency of gold is $2.44 \times 10^{-5}$, which is about 5 times that of TiN. To emit the same number of electrons as gold, we need to use 5 times the laser intensity to excite TiN.

When the two-photon photoemission was included, the response time of TiN excited by long wavelength laser was characterized again by a comparison with gold. This was because we had no autocorrelator or the second-harmonic generation crystal could work in the ultraviolet spectral region and hence the pulse duration used in the experiment was not measured accurately. We measured the pulse duration at $310 \mathrm{~nm}$ by interferometric autocorrelation and intensity autocorrelation with TiN and gold cathodes, respectively, and obtained the data described in Figs. 8 and 9. For the convenience of comparison, the data in Figs. 8 and 9 are normalized to the photocurrent with infinite time delay after removing the uniform background. The laser pulse duration fitted from the interferometric autocorrelation data was $140 \mathrm{fs}(\mathrm{Au})$ and $131 \mathrm{fs}(\mathrm{TiN})$, while the laser pulse duration obtained from intensity autocorrelation was $180 \mathrm{fs}(\mathrm{Au})$ and $173 \mathrm{fs}(\mathrm{TiN})$. The laser pulse duration measured by the two kinds of cathodes was very close, which indicated that the response time of TiN is very close to that of gold. Therefore, the two-photon photoemission from TiN is prompt.

The main reason why the pulse duration obtained by the intensity autocorrelation and interferometric autocorrelation measurement was different may be that the laser source was turned off and on again between the two measurements and the actual pulse duration was not confirmed with an interferometer. The second is the addition of the quartz wave plate, whose dispersion would broaden the laser pulse duration. Finally, there was still a small part of interferometric in the intensity autocorrelation measurement, which may be caused by the fact that the polarization directions of the two sub-beams were not completely perpendicular to each other. This may also lead to some measurement errors. 


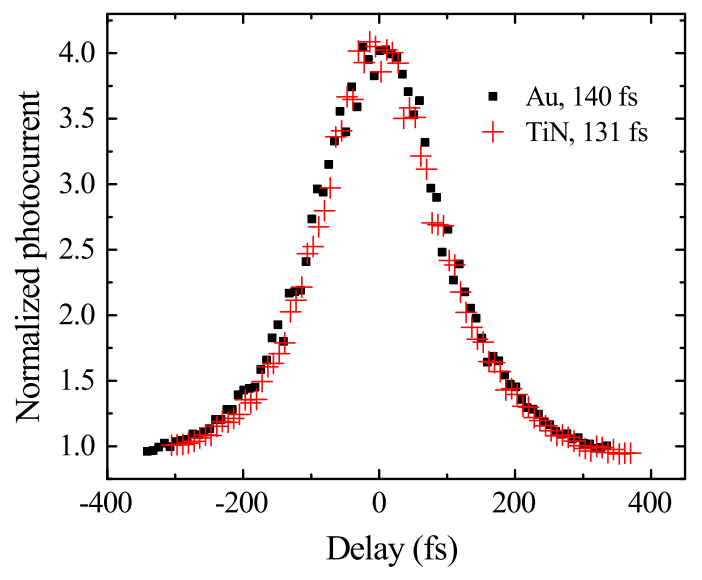

FIG. 8. Normalized interferometric autocorrelation of excited laser pulses using two-photon photoemission from an $\mathrm{Au}$ and $\mathrm{TiN}$ photocathode at a wavelength of $310 \mathrm{~nm}$. The normalized photocurrent was the average of the upper and lower envelopes of the interferometric autocorrelation signal and was normalized to the photocurrent when the time delay tends to infinity. The laser intensity of each sub-beam incident on the photocathodes was about $130 \mathrm{GW} / \mathrm{m}^{2}$.

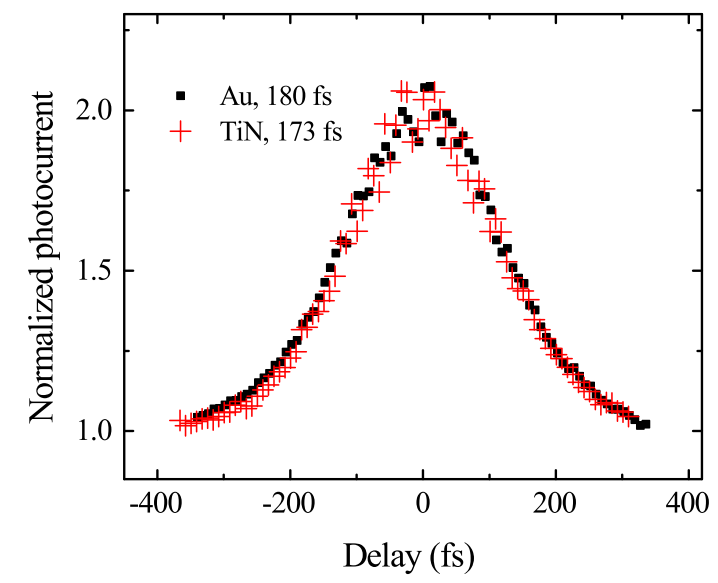

FIG. 9. Normalized intensity autocorrelation of excited laser pulses using two-photon photoemission from an $\mathrm{Au}$ and $\mathrm{TiN}$ photocathode at a wavelength of $310 \mathrm{~nm}$. The data was normalized to the photocurrent when the time delay tends to infinity. Because the reflectance of the beam splitter to the two types of polarized laser was different, the laser intensity of s-polarized sub-beam incident on the photocathodes was about $300 \mathrm{GW} / \mathrm{m}^{2}$, while p-polarized sub-beam was about $170 \mathrm{GW} / \mathrm{m}^{2}$. This led to different photocurrent density, but it did not affect the result of pulse width.

\section{CONCLUSION}

The photoemission properties of a TiN photocathode irradiated by ultraviolet pulsed laser were investigated experimentally. When the photon energy was higher than the work function, the photoemission was linear, and the intrinsic emittance was close to that of metal photocathodes, but the quantum efficiency was lower. By comparing the transverse expansion of electron bunches emitted from the TiN and gold cathodes caused by the space charge force, the response time of TiN was proven prompt. When the photon energy was slightly lower than the work function, the photoemission of TiN was nonlinear, and the intrinsic emittance increased with laser intensity. The excitation laser pulse duration was measured by the autocorrelation technique using the nonlinear photoemission of gold and TiN. The consistent pulse duration measurement results showed that the two-photon photoemission response times of TiN and gold were approximately the same.

In terms of intrinsic emittance and response time, TiN is applicable to photoemission electron sources for low emittance and short pulses. However, the quantum efficiency is very low, which limits the practicality of the TiN photocathode. Further research is needed to improve the quantum efficiency. Since the surface of our sample was not treated, that is to say, impurities and oxides may be adsorbed on the surface, future work will include surface cleaning. Utilizing microstructured and nanostructured surfaces to enhance light absorption and electron emission is also a next step for TiN is a surface plasmonic material in the near-infrared and visible spectral regions. In addition, as a reference, the photoemission characteristics of planar TiN samples excited by infrared and visible light remain yet to be investigated.

\section{ACKNOWLEDGMENTS}

This work is supported by the National Natural Science Foundation of China (Grants No. 11327902 and No. 11605001).

\section{APPENDIX A: AFM IMAGE OF TiN PHOTOCATHODE}

Surface morphology of the TiN film was measured using atomic force microscopy (Bruker Dimension Icon).

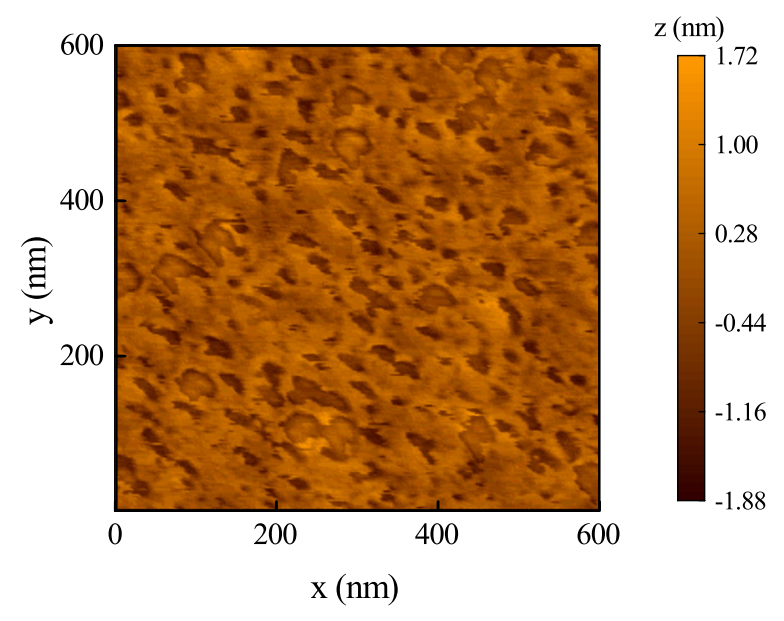

FIG. 10. AFM topography image of a TiN photocathode. The rms roughness is about $0.5 \mathrm{~nm}$. 
Figure 10 shows the AFM image of a square area of $600 \mathrm{~nm}$ by $600 \mathrm{~nm}$ on the TiN photocathode surface. A rms roughness of about $0.5 \mathrm{~nm}$ was extracted from this area.

\section{APPENDIX B: DERIVATION OF THE FITTING EXPRESSION FOR THE AUTOCORRELATION MEASUREMENT}

For the $\operatorname{sech}^{2}$-shaped pulse used in the experiment, the temporal dependence of the electric field can be expressed as

$$
E(t)=E_{p} \operatorname{sech}\left(\frac{1.763}{\tau_{p}} t\right) \exp \left\{i\left[\omega_{0} t+\phi(t)\right]\right\},
$$

where $E_{p}$ is the peak electric field, $t$ is the time, $\tau_{p}$ is the pulse duration (FWHM), $\omega_{0}$ is the angular frequency, and $\phi(t)$ is the time-dependent phase, which is a constant $\phi$ for unchirped pulses. The laser intensity is defined as

$$
I_{l}(t)=|E(t)|^{2} .
$$

The two-photon photoemission current is

$$
I_{2} \propto \int_{-\infty}^{\infty}\left[I_{l}(t)\right]^{2} d t
$$

The integral range in the above expression is over a single laser pulse. Because the response time of the current measurement device is much longer than the laser pulse length, the measured current is actually the average of the laser pulse.

For an interferometric autocorrelation, the two-photon photoemission current is

$$
\begin{aligned}
I_{2}(\tau) \propto & \int_{-\infty}^{\infty}\left[|E(t)+E(t-\tau)|^{2}\right]^{2} d t \\
= & 2 \int_{-\infty}^{\infty}\left[|E(t)|^{2}\right]^{2} d t+4 \int_{-\infty}^{\infty}|E(t)|^{2}|E(t-\tau)|^{2} d t \\
& +2 \int_{-\infty}^{\infty} \operatorname{Re}\left\{\left[E(t) E^{*}(t-\tau)\right]^{2}\right\} d t \\
& +4 \int_{-\infty}^{\infty}\left[|E(t)|^{2}+|E(t-\tau)|^{2}\right] \operatorname{Re}\left[E(t) E^{*}(t-\tau)\right] d t,
\end{aligned}
$$

where $\tau$ is the time delay. Substituting Eq. (B1) into Eq. (B4), and taking $\phi(t)$ as a constant, then carrying out the integration, one finds

$$
\begin{aligned}
I_{2}(\tau) \propto & 1+\left[6+3 \cos \left(2 \omega_{0} \tau\right)\right] \frac{\tau_{s} \cosh \left(\tau_{s}\right)-\sinh \left(\tau_{s}\right)}{\left[\sinh \left(\tau_{s}\right)\right]^{3}} \\
& +3 \cos \left(\omega_{0} \tau\right) \frac{\sinh \left(2 \tau_{s}\right)-2 \tau_{s}}{\left[\sinh \left(\tau_{s}\right)\right]^{3}}
\end{aligned}
$$

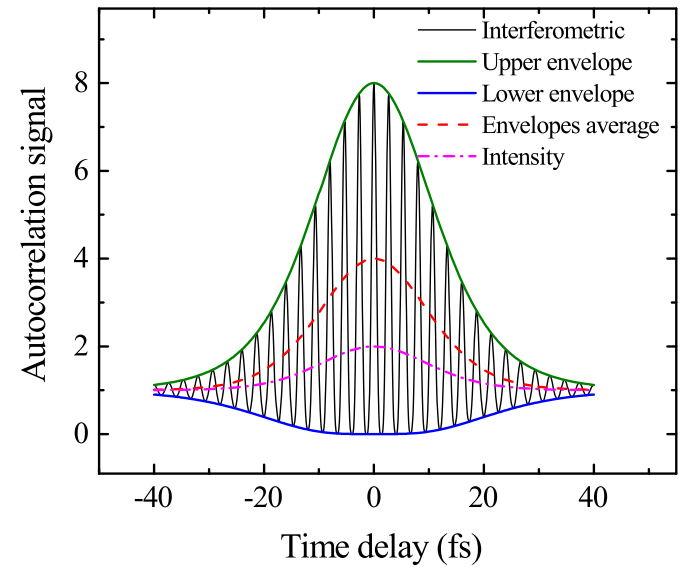

FIG. 11. Second-order autocorrelation of an $800 \mathrm{~nm} 15 \mathrm{fs}$ pulse.

where $\tau_{s}=1.763 \tau / \tau_{p}$. The right side expression is plotted in Fig. 11 as the interferometric autocorrelation curve.

From Eq. (B5), we know that the oscillation period of the second-order interferometric autocorrelation signal is the optical period. Because the time delay is changed by moving the reflective mirror, the autocorrelation signal is extremely sensitive to the vibration of the mirror. For the $310 \mathrm{~nm}$ laser, the autocorrelation signal may be an arbitrary value between the upper and lower envelopes as long as the mirror shakes back and forth by up to $155 \mathrm{~nm}$, which is difficult to prevent in the experiment. The envelopes of two-photon photoemission current are

$I_{2 \mathrm{Env}}(\tau) \propto 1+9 \frac{\tau_{s} \cosh \left(\tau_{s}\right)-\sinh \left(\tau_{s}\right)}{\left[\sinh \left(\tau_{s}\right)\right]^{3}} \pm 3 \frac{\sinh \left(2 \tau_{s}\right)-2 \tau_{s}}{\left[\sinh \left(\tau_{s}\right)\right]^{3}}$.

Taking the average of the upper and lower envelopes, we obtain

$$
I_{2 \mathrm{AveEnv}}(\tau) \propto 1+9 \frac{\tau_{s} \cosh \left(\tau_{s}\right)-\sinh \left(\tau_{s}\right)}{\left[\sinh \left(\tau_{s}\right)\right]^{3}} .
$$

For a pure two-photon process, Eq. (B6) or Eq. (B7) can be used to fit the dependence of the photocurrent on the time delay and hence extract the pulse duration. Here the photoemission contains single- and two-photon processes. The single-photon photoemission current is

$$
\begin{aligned}
I_{1}(\tau) & \propto \int_{-\infty}^{\infty}|E(t)+E(t-\tau)|^{2} d t \\
& =2 \int_{-\infty}^{\infty}|E(t)|^{2} d t+2 \int_{-\infty}^{\infty} \operatorname{Re}\left[E(t) E^{*}(t-\tau)\right] d t .
\end{aligned}
$$

Substituting Eq. (B1) into Eq. (B8), and taking $\phi(t)$ as a constant, then carrying out the integration, one finds 


$$
I_{1}(\tau) \propto 1+\frac{\tau_{s}}{\sinh \left(\tau_{s}\right)} \cos \left(\omega_{0} \tau\right)
$$

Therefore, the envelopes of single-photon photoemission current are

$$
I_{1 \mathrm{Env}}(\tau) \propto 1 \pm \frac{\tau_{s}}{\sinh \left(\tau_{s}\right)}
$$

Clearly, the average of the envelopes is a constant.

Therefore, in the interferometric autocorrelation experiment, the movable mirror was left to stay at the same time delay for a long time, and multiple signal values were recorded. The maximum and minimum of these values were taken as the upper and lower envelopes. On the basis of Eq. (B7), the average of the envelopes as a function of the time delay was fitted by

$$
\begin{aligned}
I_{\text {AveEnv }}(\tau)= & A \frac{\frac{1.763\left(\tau-\tau_{0}\right)}{\tau_{p}} \cosh \left(\frac{1.763\left(\tau-\tau_{0}\right)}{\tau_{p}}\right)-\sinh \left(\frac{1.763\left(\tau-\tau_{0}\right)}{\tau_{p}}\right)}{\left[\sinh \left(\frac{1.763\left(\tau-\tau_{0}\right)}{\tau_{p}}\right)\right]^{3}} \\
& +B,
\end{aligned}
$$

where $A, B, \tau_{0}$, and $\tau_{p}$ are the fit parameters. The appearance of $\tau_{0}$ is explained by the need for zero delay in the experiment to be determined by subsequent fitting, that is, $\tau=\tau_{0}$ means the two pulses coincide in time.

Because of the uncontrollable oscillation, it is not easy to obtain the accurate maximum and minimum in the interferometric autocorrelation measurement. Therefore, an intensity autocorrelation measurement is considered. In the intensity autocorrelation experiment, the two-photon photoemission current is

$$
\begin{aligned}
I_{i 2}(\tau) & \propto \int_{-\infty}^{\infty}\left[I_{l}(t)+I_{l}(t-\tau)\right]^{2} d t \\
& =2 \int_{-\infty}^{\infty}\left[I_{l}(t)\right]^{2} d t+2 \int_{-\infty}^{\infty} I_{l}(t) I_{l}(t-\tau) d t \\
& =2 \int_{-\infty}^{\infty}\left[|E(t)|^{2}\right]^{2} d t+2 \int_{-\infty}^{\infty}|E(t)|^{2}|E(t-\tau)|^{2} d t .
\end{aligned}
$$

Substituting Eq. (B1) into Eq. (B12) and carrying out the integration, we get the intensity autocorrelation signal:

$$
I_{i 2}(\tau) \propto 1+3 \frac{\tau_{s} \cosh \left(\tau_{s}\right)-\sinh \left(\tau_{s}\right)}{\left[\sinh \left(\tau_{s}\right)\right]^{3}}
$$

The single-photon photoemission current is independent of $\boldsymbol{\tau}$ for the intensity autocorrelation measurement. Therefore, we can still use Eq. (B11) to fit the photoemission current as a function of the time delay in the intensity autocorrelation experiment. Although the mirror vibration did not cause serious fluctuation of the photocurrent in the intensity autocorrelation measurement, the photocurrent data with the same time delay were collected continuously over a period of time and averaged in order to obtain accurate measurement results.

[1] A. H. Zewail, 4d ultrafast electron diffraction, crystallography, and microscopy, Annu. Rev. Phys. Chem. 57, 65 (2006).

[2] P. Musumeci, J. T. Moody, C. M. Scoby, M. S. Gutierrez, H. A. Bender, and N. S. Wilcox, High quality single shot diffraction patterns using ultrashort megaelectron volt electron beams from a radio frequency photoinjector, Rev. Sci. Instrum. 81, 013306 (2010).

[3] P. Emma, R. Akre, J. Arthur, R. Bionta, C. Bostedt, J. Bozek, A. Brachmann, P. Bucksbaum, R. Coffee, F.-J. Decker et al., First lasing and operation of an angstromwavelength free-electron laser, Nat. Photonics 4, 641 (2010).

[4] W. S. Graves, W. Brown, F. X. Kaertner, and D. E. Moncton, MIT inverse Compton source concept, Nucl. Instrum. Methods Phys. Res., Sect. A 608, S103 (2009).

[5] T. van Oudheusden, E. F. de Jong, S. B. van der Geer, W. P. E. M. Op't Root, O. J. Luiten, and B. J. Siwick, Electron source concept for single-shot sub-100 fs electron diffraction in the $100 \mathrm{keV}$ range, J. Appl. Phys. 102, 093501 (2007).

[6] W. A. Barletta, J. Bisognano, J. N. Corlett, P. Emma, Z. Huang, K.-J. Kim, R. Lindberg, J. B. Murphy, G. R. Neil, D. C. Nguyen et al., Free electron lasers: Present status and future challenges, Nucl. Instrum. Methods Phys. Res., Sect. A 618, 69 (2010).

[7] D. H. Dowell, I. Bazarov, B. Dunham, K. Harkay, C. Hernandez-Garcia, R. Legg, H. Padmore, T. Rao, J. Smedley, and W. Wan, Cathode R\&D for future light sources, Nucl. Instrum. Methods Phys. Res., Sect. A 622 , 685 (2010).

[8] L. Cultrera, Cathodes for photoemission guns, in Proceedings of the 24th Particle Accelerator Conference, PAC2011, New York, 2011 (IEEE, New York, 2011), p. 2099.

[9] R. Xiang and J. Teichert, Photocathodes for high brightness photoinjectors, Phys. Procedia 77, 58 (2015).

[10] A. H. Sommer, Photoemissive Materials: Preparation, Properties and Uses (John Wiley \& Sons, Inc., New York, 1968), pp. 5-9.

[11] S. H. Kong, J. Kinross-Wright, D. C. Nguyen, and R. L. Sheffield, Photocathodes for free electron lasers, Nucl. Instrum. Methods Phys. Res., Sect. A 358, 272 (1995).

[12] P. Musumeci, J. G. Navarro, J. B. Rosenzweig, L. Cultrera, I. Bazarov, J. Maxson, S. Karkare, and H. Padmore, Advances in bright electron sources, Nucl. Instrum. Methods Phys. Res., Sect. A 907, 209 (2018).

[13] D. H. Dowell and J. F. Schmerge, Quantum efficiency and thermal emittance of metal photocathodes, Phys. Rev. ST Accel. Beams 12, 074201 (2009). 
[14] P. Musumeci, L. Cultrera, M. Ferrario, D. Filippetto, G. Gatti, M. S. Gutierrez, J. T. Moody, N. Moore, J. B. Rosenzweig, C. M. Scoby et al., Multiphoton Photoemission from a Copper Cathode Illuminated by Ultrashort Laser Pulses in an RF Photoinjector, Phys. Rev. Lett. 104, 084801 (2010).

[15] R. K. Li, H. To, G. Andonian, J. Feng, A. Polyakov, C. M. Scoby, K. Thompson, W. Wan, H. A. Padmore, and P. Musumeci, Surface-Plasmon Resonance-Enhanced Multiphoton Emission of High-Brightness Electron Beams from a Nanostructured Copper Cathode, Phys. Rev. Lett. 110, 074801 (2013).

[16] A. Polyakov, C. Senft, K. F. Thompson, J. Feng, S. Cabrini, P. J. Schuck, H. A. Padmore, S. J. Peppernick, and W. P. Hess, Plasmon-Enhanced Photocathode for High Brightness and High Repetition Rate X-Ray Sources, Phys. Rev. Lett. 110, 076802 (2013).

[17] H. Li, C. Limborg-Deprey, C. Adolphsen, D. McCormick, M. Dunning, K. Jobe, T. Raubenheimer, A. Vrielink, T. Vecchione, F. Wang et al., Two-photon photoemission from a copper cathode in an X-band photoinjector, Phys. Rev. Accel. Beams 19, 023401 (2016).

[18] S. Karkare and I. Bazarov, Effects of surface nonuniformities on the mean transverse energy from photocathodes, Phys. Rev. Applied 4, 024015 (2015).

[19] J. Maxson, P. Musumeci, L. Cultrera, S. Karkare, and H. Padmore. Ultrafast laser pulse heating of metallic photocathodes and its contribution to intrinsic emittance, Nucl. Instrum. Methods Phys. Res., Sect. A 865, 99 (2017).

[20] D. Shah, H. Reddy, N. Kinsey, V. M. Shalaev, and A. Boltasseva, Optical properties of plasmonic ultrathin TiN films, Adv. Opt. Mater. 5, 1700065 (2017).

[21] Y. Liu, S. Kijima, E. Sugimata, M. Masahara, K. Endo, T. Matsukawa, K. Ishii, K. Sakamoto, T. Sekigawa, H. Yamauchi et al., Investigation of the TiN gate electrode with tunable work function and its application for FinFET fabrication, IEEE Trans. Nanotechnol. 5, 723 (2006).

[22] Y. Liu, T. Hayashida, T. Matsukawa, K. Endo, M. Masahara, S. O’uchi, K. Sakamoto, K. Ishii, J. Tsukada, Y. Ishikawa et al., Nitrogen gas flow ratio and rapid thermal annealing temperature dependences of sputtered titanium nitride gate work function and their effect on device characteristics, Jpn. J. Appl. Phys. 47, 2433 (2008).

[23] G. V. Naik, J. L. Schroeder, X. Ni, A. V. Kildishev, T. D. Sands, and A. Boltasseva, Titanium nitride as a plasmonic material for visible and near-infrared wavelengths, Opt. Mater. Express 2, 478 (2012).

[24] G. V. Naik, V. M. Shalaev, and A. Boltasseva, Alternative plasmonic materials: Beyond gold and silver, Adv. Mater. 25, 3264 (2013).

[25] A. Catellani and A. Calzolari, Plasmonic properties of refractory titanium nitride, Phys. Rev. B 95, 115145 (2017).

[26] S. Murai, K. Fujita, Y. Daido, R. Yasuhara, R. Kamakura, and K. Tanaka, Plasmonic arrays of titanium nitride nanoparticles fabricated from epitaxial thin films, Opt. Express 24, 1143 (2016).

[27] W. Guo, R. Mishra, C. Cheng, B. Wu, L. Chen, M. Lin, and S. Gwo, Titanium nitride epitaxial films as a plasmonic material platform: alternative to gold, ACS Photonics 6, 1848 (2019).

[28] J. Pflüger, J. Fink, W. Weber, and K.-P. Bohnen, Dielectric properties of $\mathrm{TiC}_{x}, \mathrm{TiN}_{x}, \mathrm{VC}_{x}$, and $\mathrm{VN}_{x}$ from 1.5 to $40 \mathrm{eV}$ determined by electron-energy-loss spectroscopy, Phys. Rev. B 30, 1155 (1984).

[29] V. Schnabel, R. Spolenak, M. Doebeli, and H. Galinski, Structural color sensors with thermal memory: Measuring functional properties of Ti-based nitrides by eye, Adv. Opt. Mater. 6, 1800656 (2018).

[30] A. Torgovkin, S. Chaudhuri, A. Ruhtinas, M. Lahtinen, T. Sajavaara, and I. J. Maasilta, High quality superconducting titanium nitride thin film growth using infrared pulsed laser deposition, Supercond. Sci. Technol. 31, 055017 (2018).

[31] D. Janssen, A. Arnold, H. Büttig, U. Lehnert, P. Michel, P. Murcek, C. Schneider, R. Schurig, F. Staufenbiel, J. Teichert et al., Review on superconducting rf guns, in Proceedings of the 12th International Workshop on RF Superconductivity, Cornell University, Ithaca, New York, 2005, edited by S. Belomestnykh, M. Liepe, and H. Padamsee (Cornell University, Ithaca NY, 2005), p. 433.

[32] C. An, R. Zhu, J. Xu, Y. Liu, X. Hu, J. Zhang, and D. Yu, Increase of intrinsic emittance induced by multiphoton photoemission from copper cathodes illuminated by femtosecond laser pulses, AIP Adv. 8, 055225 (2018).

[33] T. Vecchione, I. Ben-Zvi, D. H. Dowell, J. Feng, T. Rao, J. Smedley, W. Wan, and H. A. Padmore, A low emittance and high efficiency visible light photocathode for high brightness accelerator-based x-ray light sources, Appl. Phys. Lett. 99, 034103 (2011).

[34] H. Lee, S. Karkare, L. Cultrera, A. Kim, and I. V. Bazarov, Review and demonstration of ultra-low-emittance photocathode measurements, Rev. Sci. Instrum. 86, 073309 (2015).

[35] T. Vecchione, D. Dowell, W. Wan, J. Feng, and H. A. Padmore, Quantum efficiency and transverse momentum from metals, in Proceedings of FEL2013, New York, NY, 2013 (JACoW, Geneva, Switzerland, 2013), p. 424.

[36] E. P. Ippen and C. V. Shank, in Ultrashort Light Pulses, edited by S. L. Shapiro (Springer, New York, 1977), pp. 83-85.

[37] R. Trebino, Frequency-Resolved Optical Gating: The Measurement of Ultrashort Laser Pulses (Springer, New York, 2000), pp. 84-85.

[38] J.-C. Diels and W. Rudolph, Ultrashort Laser Pulse Phenomena Fundamentals, Techniques, and Applications on a Femtosecond Time Scale, 2nd ed. (Academic Press, San Diego, 2006), pp. 466-468.

[39] T. Tsang, T. Srinivasan-Rao, and J. Fischer, Surfaceplasmon field-enhanced multiphoton photoelectric emission from metal films, Phys. Rev. B 43, 8870 (1991).

[40] T. Tsang, Measurement of femtosecond electron bunches from metal photocathodes, Appl. Phys. Lett. 63, 871 (1993).

[41] C. P. Hauri, R. Ganter, F. Le Pimpec, A. Trisorio, C. Ruchert, and H. H. Braun, Intrinsic Emittance Reduction of an Electron Beam from Metal Photocathodes. Phys. Rev. Lett. 104, 234802 (2010).

[42] M. C. Divall, E. Prat, S. Bettoni, C. Vicario, A. Trisorio, T. Schietinger, and C. P. Hauri, Intrinsic emittance reduction 
of copper cathodes by laser wavelength tuning in an $\mathrm{rf}$ photoinjector, Phys. Rev. ST Accel. Beams 18, 033401 (2015).

[43] E. Prat, S. Bettoni, H.-H. Braun, R. Ganter, and T. Schietinger, Measurements of copper and cesium telluride cathodes in a radio-frequency photoinjector, Phys. Rev. ST Accel. Beams 18, 043401 (2015).

[44] L. Cultrera, C. Ristoscu, G. Gatti, P. Miglietta, F. Tazzioli, and A. Perrone, Photoemission characteristics of PLD grown $\mathrm{Mg}$ films under UV laser irradiation, J. Phys. D 40, 5965 (2007).

[45] H. J. Qian, J. B. Murphy, Y. Shen, C. X. Tang, and X. J. Wang, Surface photoemission in a high-brightness electron beam radio frequency gun, Appl. Phys. Lett. 97, 253504 (2010).

[46] R. Xiang, A. Arnold, P. Lu, P. Murcek, J. Teichert, and H. Vennekate, Study of magnesium photocathodes for superconducting rf photoinjectors, in Proceedings of the 9th International Particle Accelerator Conference, Vancouver, BC, Canada, 2018 (JACoW, Geneva, Switzerland, 2018), p. 4142.
[47] F. Zhou, J. C. Sheppard, T. Vecchione, E. Jongewaard, A. Brachmann, J. Corbett, S. Gilevich, and S. Weathersby, Establishing reliable good initial quantum efficiency and in situ laser cleaning for the copper cathodes in the rf gun, Nucl. Instrum. Methods Phys. Res., Sect. A 783, 51 (2015).

[48] D. H. Dowell, F. K. King, R. E. Kirby, J. F. Schmerge, and J. M. Smedley, In situ cleaning of metal cathodes using a hydrogen ion beam, Phys. Rev. ST Accel. Beams 9, 063502 (2006).

[49] R. Valizadeh, A. N. Hannah, K. J. Middleman, B. L. Militsyn, T. C. Q. Noakes, M. D. Roper, and R. Santer, Preparation of the polycrystalline copper photocathodes for vela rf photocathode gun, in Proceedings of the 4th International Particle Accelerator Conference, IPAC2013, Shanghai, China, 2013 (JACoW, Shanghai, China, 2013), p. 440.

[50] J. Feng, J. Nasiatka, W. Wan, S. Karkare, J. Smedley, and H. A. Padmore, Thermal limit to the intrinsic emittance from metal photocathodes, Appl. Phys. Lett. 107, 134101 (2015). 